Revista Colombiana de Obstetricia y Ginecología Vol. 55 No.4 • 2004 • (287-292)

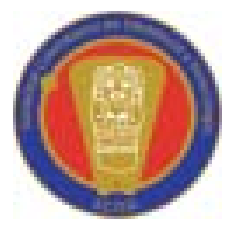

InVESTIGACión Original

\title{
EFECTO DEL HÁBITO DE FUMAR SOBRE LOS RESULTADOS DE LABORATORIO DE LA FERTILIZACIÓN IN VITRO
}

\author{
EFFECT OF CIGARETTE SMOKING ON LABORATORIAL OUTCOME OF \\ IN VITRO FERTILIZATION \\ INFLUIÊNCIA DO TABAGISMO NOS RESUILTADOS LABORATORIAIS \\ DA FERTILIZAÇÃO IN VITRO
}

\author{
Adriana Arent, M.D., M. Sc.*, Mariangela Badalotti, M.D., M. Sc.**, \\ Julia Machado***, Rafaella Petracco****, Alvaro Petracco, M.D.***** \\ Recibido: agosto 27/04 - Revisado: noviembre 8/04 - Aceptado: noviembre 22/04
}

\section{RESUIMEN}

Objetivo: evaluar la influencia del tabaquismo femenino, activo y pasivo, sobre los resultados de la fertilización in vitro (FIV) de mujeres fumadoras.

Diseño: estudio de cohorte.

Materiales y métodos: ciento diecinueve (119) parejas fueron sometidas a FIV, en primer intento, cuya indicación fue el factor tubario, fueron divididos en 3 grupos: mujeres no fumadoras $(n=81)$, mujeres fumadoras pasivas $(n=15)$ y mujeres fumadoras verdaderas $(n=23)$. Los resultados de laboratorio de la FIV fueron analizados en separado.

Resultados: las mujeres fumadoras tuvieron un bajo índice de fertilización $(40,7 \%)$ y mala calidad embrionaria $(70,6 \%)$ en comparación con las no fumadoras $(59,1 \%$ y $82,5 \%)$ y las fumadoras

\footnotetext{
Médica Ginecologista. Preceptora Mestre do Serviço de Ginecologia do HSL/PUCRS. Membro do Fertilitat - Centro de Medicina Reprodutiva.

** Médica Ginecologista. Professora Mestre da Faculdade de Medicina PUCRS. Chefe do Serviço de Ginecologia do HSL/PUCRS. Diretora do Fertilitat - Centro de Medicina Reprodutiva.

*** Acadêmica da Faculdade de Medicina da PUCRS.

**** Acadêmica da Faculdade de Medicina da ULBRA.

***** Médico Ginecologista. Professor da Faculdade de Medicina PUCRS Diretor do Fertilitat - Centro de Medicina Reprodutiva.

Trabajo realizado nel Fertilitat - Centro de Medicina Reprodutiva. Porto Alegre, Brasil.

Correspondência: Adriana Arent. Quintino Bocaiúva, 1617/301, Porto Alegre, RS Brasil. CEP:90440-051. e-mail: adriarent@ig.com.br - fertilitat@fertilitat.com.br
}

pasivas $(67,4 \%$ y $87,9 \%)(p<0,05)$. No se ha encontrado diferencia en el numero de oocitos obtenidos y de embriones clivados entre los grupos.

Conclusiones: los resultados indican que el éxito de la FIV puede estar afectado negativamente en las fumadoras, donde el índice de fertilización es menor y la calidad embrionaria peor.

Palabras clave: tabaco, tabaquismo, nicotina, infertilidad, FIV

\section{SUMMARY}

Objective: to evaluate the influence of female cigarette smoking on laboratory results of in-vitro fertilization (IVF).

Design: cohort study.

Patients and methods: a total of 119 infertile couples, who were submitted to the first cycle of IVF due to tubal factor, were divided in three groups: non-smokers women $(n=81)$, passive smokers women $(n=15)$ and active smokers women $(n=23)$. The laboratorial results of IVF were analyzed among the sub-groups.

Results: women who smoked had a lower fertilization rate $(40.7 \%)$ and a worse embryo quality (70.6\%) than the non-smokers $(59 \%$ and $81.3 \%)$ and the passive smokers $(67.4 \%$ and $87.9 \%)(p<0.05)$. There were no significant 
differences on the number of oocytes retrieved and embryo cleavage rate between the different groups.

Conclusions: the results of this study indicate that the success of IVF can be affected negatively in the smoker women, due to smaller fertilization rate and the worse embryonic quality.

Key words: tobacco, smoking, nicotine, infertility, IVF

\section{INTRODUÇÃO}

Usado inicialmente nas Américas, com propósitos religiosos e em cerimônias, o tabaco, em 1942, começou a ser levado para a Europa. Hoje, um terço da população mundial de 15 anos ou mais é fumante, o que corresponde a 1,2 bilhões de pessoas. ${ }^{1}$ A prevalência do tabagismo entre homens tem se mantido em torno de $50 \%$, enquanto que a prevalência entre as mulheres tem aumentado substancialmente nos últimos 20 anos, marcadamente entre as mais jovens. $\mathrm{Na}$ Inglaterra, aos 15 anos de idade 33\% das meninas são tabagistas. ${ }^{2}$ Dados nacionais recentes têm mostrado prevalência ao redor de $40 \%$ entre homens e $25 \%$ entre mulheres. ${ }^{3}$

Os efeitos negativos do consumo de cigarros sobre a saúde geral são bem conhecidos, mas o tabagismo pode afetar também a fertilidade. A nicotina e outros agentes derivados do tabaco podem atuar ao nível de hipotálamo e hipófise, ovários, trompas de falópio, oócitos, embriões e endométrio. ${ }^{4-11}$ Nas mulheres o tabagismo está associado com diminuição dos níveis circulantes de estrogênio ${ }^{5}$ e menopausa antecipada. ${ }^{12} \mathrm{O}$ consumo de cigarros pode diminuir a mobilidade e a função ciliar do epitélio da tuba, justificando a base biológica para a associação entre o tabagismo e a doença inflamatória pélvica. ${ }^{13}$ Além disso, pode aumentar o risco de infertilidade devida ao fator cervical, possivelmente secundário ao fato de que o muco cervical de fumantes contém componentes do tabaco que podem ser tóxicos aos espermatozóides. ${ }^{14,15}$
Quanto à reprodução assistida, a nicotina e seus metabólitos podem determinar redução do número de folículos, diminuição das taxas de fertilização e gestação nos tratamentos de fertilização in vitro (FIV), e aumentar a taxa de abortamento. ${ }^{16}$ Mas os resultados da literatura são conflitantes e muitas vezes a exposição passiva ao cigarro não é levada em consideração. A maioria dos estudos mostra que mulheres tabagistas apresentam sucesso menor nos procedimentos de FIV. ${ }^{17,18}$. A reposta ovariana diminuída nas pacientes tabagistas tem sido apontada como uma das principais razões para o resultado inferior da FIV em tabagistas.

Este estudo avalia o impacto do tabagismo feminino (ativo e passivo) sobre a taxa de recuperação oocitária e sobre os resultados laboratoriais (taxa de fertilização, taxa de clivagem e qualidade embrionária) em um programa de FIV.

\section{PACIENTES E MÉTODOS}

Desenho do estudo: estudo de coorte.

Pacientes: foram avaliados 119 casais inférteis que estavam realizando o primeiro ciclo de FIV. Os critérios de inclusão para este estudo foram: a) fator tubário como causa da infertilidade; b) espermograma normal e c) folículo-aspiração após a estimulação hormonal. As pacientes foram divididas em três grupos de acordo com o tipo de exposição ao tabaco: A) tabagistas $(n=23)$, B) tabagistas passivas $(n=15)$ e $C)$ não tabagistas $(n=81)$. Os critérios de exclusão foram história de cirurgia ovariana (ooforoplastia, ooforectomia) e tabagismo no passado.

A história de tabagismo foi coletada através de questionário preenchido pelos casais durante consulta médica. Mulheres que nunca fumaram ou foram consideradas não-tabagistas, enquanto que as que fumavam diariamente foram consideradas tabagistas. O grupo de tabagistas passivas foi constituído por pacientes não tabagistas cujos companheiros eram fumantes.

Local: Fertilitat - Centro de Medicina Reprodutiva. Centro privado de atendimento 
terciário, com média de 300 ciclos FIV/ano. Acreditado pela Red Latinoamericana de Reproducción Asistida. Porto Alegre, RS, Brasil.

\section{Procedimento de FIV}

As pacientes foram submetidas à estimulação ovariana com gonadotrofina menopáusica humana (hMG) após dessensibilização hipofisária com acetato de leuprolide, utilizando protocolos de indução já estabelecidos. ${ }^{19,20} \mathrm{O}$ desenvolvimento folicular foi controlado por ecografia transvaginal e foi administrado gonatotrofina coriônica humana (hCG) quando pelo menos dois folículos apresentavam $18 \mathrm{~mm}$ de diâmetro. A aspiração folicular foi realizada 35 horas após o hCG, por via transvaginal, sob visão ecográfica. ${ }^{21} \mathrm{O}$ líquido folicular aspirado era enviado imediatamente ao laboratório de reprodução assistida para screening dos oócitos. Foram inseminados os oócitos em estágio de metáfase II (M II).

A coleta seminal foi realizada por masturbação e o sêmen preparado por percoll. A inseminação foi realizada 4 horas após a coleta oocitária, utilizandose 100.000 espermatozóides móveis/oócito. A fertilização foi comprovada pela presença de dois pró-nucleos e corpo polar dividido,18 a 20 horas após a inseminação. Os oócitos fertilizados foram cultivados com meio de cultura HTF® (human tubal fluid, Irvine Scientific) suplementado com 15\% de SSS (serum substite solution, Irvine Scientific).

A divisão embrionária foi observada 24 e 48 horas após a fertilização. O grau morfológico usado para classificar os embriões foi baseado nos critérios da Rede Latinoamericana de Reprodução Assistida (Quadro 1), que utiliza os parâmetros de simetria de blastômeras e percentagem de fragmentação citoplasmática. ${ }^{22}$ Segundo esta classificação os embriões são graduados em grau I, grau II, grau III e grau IV sendo considerados os melhores embriões aqueles com graus I e II.

\section{Variáveis analisadas}

- Oócitos recuperados: número total de oócitos obtidos na aspiração folicular, em

- Oócitos recuperados por paciente: é o número oócitos recuperados dividido pelo número de pacientes.

- Óócitos inseminados: é número de oócitos que foram inseminados.

- Oócitos fertilizados: é o número de oócitos que fertilizaram.

- Taxa de fertilização: é o número de oócitos fertilizados dividido pelo número de oócitos inseminados, multiplicado por 100 (percentual).

- Embriões divididos: é o número de oócitos fertilizados que se dividiram, gerando embriões.

- Taxa de clivagem embrionária: é o número de embriões divididos dividido pelo número de oócitos fertilizados, multiplicado por 100 (percentual).

- Qualidade embrionária: é uma classificação embrionária, para a qual foram utilizados os critérios da Red Latinoamericana de Reproducción Asistida (RED) (Quadro 1).

\section{Análise estatística:}

Neste estudo foram avaliados os resultados laboratoriais da IVF em 3 grupos de pacientes, divididos segundo a exposição ao tabaco. $\mathrm{O}$ número de oócitos recuperados por paciente foi analisado pelo teste de Anova. As taxas de fertilização oocitária, de clivagem embrionária e de qualidade

Quadro 1- Graduação morfológica para embrióes humanos cultivados in vitro. Critérios da RED.

\begin{tabular}{ll} 
GRAU & DESCRIÇÃO \\
\hline Grau I & $\begin{array}{l}\text { Embriões com blastômeras simétricas e } \\
\text { ausência de fragmentação citoplasmática }\end{array}$ \\
\hline Grau II & $\begin{array}{l}\text { Embriões com blastômeras simétricas ou } \\
\text { assimétricas e/ou com menos de 20\% } \\
\text { de fragmentação citoplasmática }\end{array}$ \\
\hline Grau III & $\begin{array}{l}\text { Embriões com blastômeras simétricas ou } \\
\text { não, e/ou com 20 a 50\% de fragmentação } \\
\text { citoplasmática }\end{array}$ \\
\hline Grau IV & $\begin{array}{l}\text { Embriões com blastômeras simétricas ou } \\
\text { não e/ou com mais de 50\% de } \\
\text { fragmentação citoplasmática }\end{array}$ \\
\hline
\end{tabular}


embrionária foram analisados utilizando teste do $\grave{a}^{2}$ Um erro á de $5 \%(\mathrm{p}<0,05)$ foi considerado significativo.

\section{Ética:}

Todas pacientes assinaram termo de consentimento informado antes do tratamento de fertilização, disponibilizando a utilização de seus dados para fins científicos; sendo este procedimento aprovado pelo Comitê de Ética em Pesquisa da Pontifícia Universidade Católica do Rio Grande do Sul. Nenhum procedimento além dos necessários ao tratamento de fertilização assistida foi realizado.

\section{RESUILTADOS}

Foi avaliado um total de 119 ciclos, dos quais 23 de mulheres tabagistas (grupo A), 15 de mulheres tabagistas passivas (grupo B) e 81 de mulheres não tabagistas (grupo C) (Tab 1). A idade média destas mulheres foi de 35,1 anos, sem diferença estatística entre os 3 grupos.

$\mathrm{O}$ tabagismo não interferiu sobre o número de oócitos recuperados por paciente (grupo $\mathrm{A}=$ $11,4$; grupo $\mathrm{B}=8,5$ e grupo $\mathrm{C}=8,6 ; \mathrm{p}>0,05)$ assim como também não afetou a taxa de clivagem embrionária (grupo $\mathrm{A}=86,1 \%$; grupo $\mathrm{B}=$ 90,6\% e grupo $C=76,4 \% ; p>0,05)$ (Tab 1).

A taxa de fertilização das pacientes tabagistas $(40,7 \%)$ foi significativamente inferior $(\mathrm{p}<0,05)$ quando comparada à de tabagistas passivas $(67,4 \%)$ e à de não tabagistas (59\%) (Tab 1).

A qualidade embrionária também foi negativamente afetada no grupo de pacientes tabagistas, onde embriões de boa qualidade totalizaram 70,6\% dos embriões clivados no grupo A, em comparação com os percentuais de $87,9 \%$ e $82,5 \%$ nos grupo B e C, respectivamente $(\mathrm{p}<0,05)($ Tab 1).

\section{DISCUSSÃO}

A literatura tem sugerido que mulheres tabagistas apresentam uma probabilidade diminuída de sucesso nos procedimentos de FIV quando comparadas àquelas não fumantes, e a reserva ovariana diminuída tem sido sugerida como o mecanismo chave deste resultado. Uma pobre resposta ao teste do clomifeno sugere diminuição de reserva ovariana e tem sido encontrada mais freqüentemente em fumantes. ${ }^{11}$ Outra medida de reserva ovariana é a dosagem de FSH na fase folicular inicial. Um nível elevado de FSH é preditivo de má resposta à indução da ovulação e piores resultados nos tratamentos de FIV e um estudo demonstrou que mulheres tabagistas apresentam níveis basais de FSH superiores ao de não fumantes. ${ }^{5,23}$ Alguns autores observaram redução do número de oócitos em pacientes fumantes submetidas a FIV, quando comparadas a não fumantes. ${ }^{24}$ No presente estudo

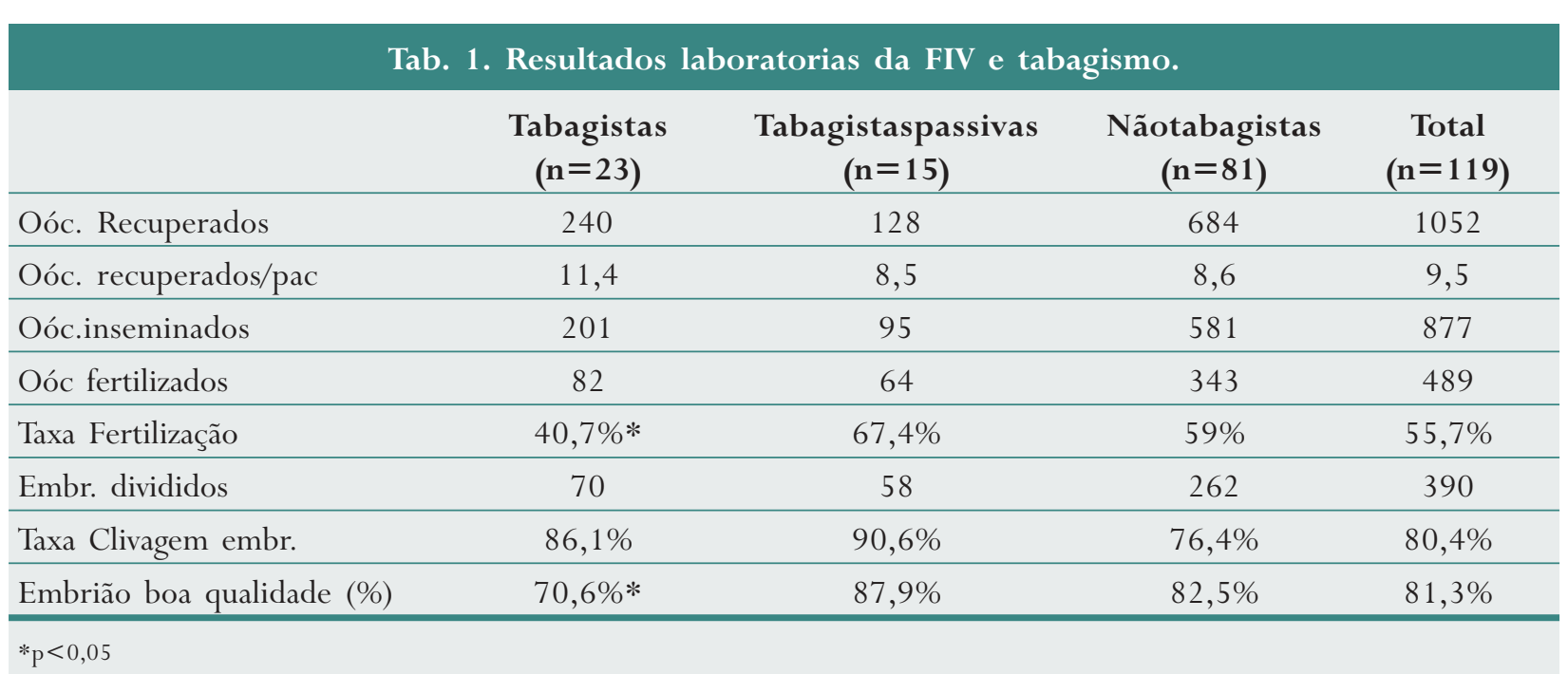


não houve diferença estatisticamente significativa no número de oócitos recuperados por pacientes tabagistas, tabagistas passivas e não tabagistas $(11,4$, 8,5 e 8,6, respectivamente). Uma possível limitação deste estudo na avaliação da resposta ovariana de pacientes fumantes é que não foi levado em consideração o tempo de exposição ao tabaco e a quantidade de cigarros e não foi avaliada a taxa de cancelamento de ciclos por má resposta ovariana.

Diminuição na taxa de fertilização em pacientes fumantes foi descrita em alguns estudos, $8,18,25$ enquanto outros encontraram resultados similares. ${ }^{26-28}$ Esta discrepância pode ser devida a diferença nos critérios de inclusão das pacientes, como idade e diferentes protocolos de estimulação ovariana. Nossos dados concordam com os estudos que evidenciam prejuízo sobre a fertilização oocitária, uma vez que a taxa de fertilização das pacientes tabagistas $(40,7 \%)$ foi significativamente inferior $(\mathrm{p}<0,05)$ quando comparadas aos outros grupos (Tab 1).

Estudos sobre a qualidade embrionária de pacientes tabagistas são escassos, sendo que a maior parte dos estudos é centrado sobre a resposta ovariana e a taxa de fertilização. Neste estudo observamos o impacto negativo do tabagismo sobre a qualidade embrionária. Esta relação foi observada somente nas pacientes tabagistas, e não nas tabagistas passivas. Apesar da taxa de divisão embrionária ter sido similar entre os grupos estudados, embriões de boa qualidade totalizaram $70,6 \%$ dos embriões das pacientes tabagistas, índice significativamente inferior $(\mathrm{p}<0.05)$ ao das pacientes tabagistas passivas $(87,9 \%)$ e ao das não tabagistas $(82,5 \%)$. Estes resultados concordam com os observados por Weigert M e cols. (23) que também observaram impacto negativo sobre a qualidade embrionária nas pacientes fumantes, atribuído ao aumento significante dos níveis de hormônio luteinizante (LH) nas pacientes tabagistas, além de menor número de oócitos ( $\mathrm{p}$ $=0,002)$.

\section{CONCLUSÃO}

Este estudo mostra que o resultado laboratorial da FIV pode ser negativamente afetado nas mulheres tabagistas, pela menor taxa de fertilização e a pior qualidade embrionária. Não observamos prejuízo nos resultados laboratoriais de pacientes tabagistas passivas, talvez devido à baixa casuística, assim como não foi demonstrada diferença de resposta ovariana entre os grupos. Outros estudos sobre do impacto do tabagismo passivo sobre os resultados da FIV devem ser realizados, com especial atenção ao tempo de exposição ao tabaco e a quantidade de cigarros. Da mesma forma sugerese estudos com maior casuísta para avaliar a interferência do tabagismo passivo, levando-se em consideração além dos hábitos do parceiro, o ambiente de trabalho.

\section{REFERÊNCIAS}

1. Yach D, Bettcher D. Globalisation of tobacco industry influence and new global responses. Tob Control 2000;9:206-16.

2. The National Public Health Institute. Smoking Prevalence and Tobacco Policies in the Member States of the European Union. Helsinki: The Institute; 1997.

3. Ministério da Saúde. Instituto Nacional do Câncer. Prevalência de Tabagismo no Brasil. Dados dos inquéritos epidemiológicos em capitais brasileiras. Rio de Janeiro: O Instituto; 2004.

4. Hughes EG, Brennan BG. Does cigarette smoking impair natural or assisted fecundity? Fertl Steril 1996;66:679-89.

5. MacMahon B, Trichopoulus D, Cole P. Cigarette smoking and urinary estrogens. $N$ Engl J Med 1982;307:1062-5.

6. Sterzik K, Strehler E, De Santo M, Trumpp N, Abt $\mathrm{M}$, Rosenbusch $\mathrm{B}$, et al. Influence of smoking on fertility in women attending an in vitro fertilization program. Fertil Steril 1996;65:810-4.

7. Barbieri RL, McShane PM, Ryan KJ. Constituents of cigarette smoke inhibit human granulosa cell aromatase. Fertil Steril 1986;46:232-6.

8. Elenbogen A, Lipitz S, Mashiach S, Dor J, Levran D, Ben-Rafael Z. The effect of smoking on the outcome 
of in-vitro fertilization-embryo transfer. Hum Reprod 1991;6:242-4.

9. Fredricsson B, Gilljam H. Smoking and reproduction. Short and long term effects and benefits of smoking cessation. Acta Obstet Gynecol Scand 1992;71:580-92.

10. Pattinson HA, Taylor PJ, Pattinson MH. The effect of cigarette smoking on ovarian function and early pregnancy outcome of in vitro fertilization treatment. Fertil Steril 1991;55:780-3.

11. Sharara FI, Beatse SN, Leonardi MR, Navot D, Scott RT Jr. Cigarette smoking acelarates the development of diminished ovarian reserve as evidenced by the clomiphene citrate chalenge test. Fertil Steril 1994;62:257-62.

12.Jick H, Porter J. Relation between smoking and age of natural menopause. Report from the Boston Collaborative Drug Surveillance Program, Boston University Medical Center. Lancet 1977;1:1354-5.

13. Neri A, Marcus SL. Effects of nicotine on the motility of the oviducts in the rhesus monkey: a preliminary report. J Reprod Fertil 1972;31:91-7.

14. Phipps WR, Cramer DW, Schiff I, Belisle S, Stillman $\mathrm{R}$, Albrecht B, et al. The association between smoking and female infertility as influenced by cause of the infertility. Fertil Steril 1987;48:377-82.

15.Sasson JM, Haley NJ, Hoffman D, Wynder EL, Hellberg D, Nilsson S. Cigarette smoking and neoplasia of the uterine cervix:smoke constituents in cervical mucus. N Engl J Med 1985;312:315-6.

16. Klonoff-Cohen HK, Natarajan L, Marss R, Yee B. Effects of female and male smoking on success rates of IVF and gamete intra-Fallopian transfer. Human Reprod 2001;16:1382-90.

17. Hughes EG, Yeo J, Claman P, YoungLai EV, Sagle MA, Daya S, et al. Cigarette smoking and the outcome of in vitro fertilization: measurement of effect size and levels of action. Fertil Steril 1994;62:807-14.

18. Rosevear SK, Holt DW, Lee TD, Ford WC, Wardle PG, Hull MG. Smoking and decreased fertilization rates in vitro. Lancet 1992;340:1195-6.
19. Neveu S, Hedon B, Bringer J, Chinchole JM, Arnal F, Humeau $\mathrm{C}$, et al. Ovarian stimulation by combination of gonadotropin-releasing hormone agonist and gonadotropins for in vitro fertilization. Fertil Steril 1987;47:639-43.

20. Scott RT Jr, Rosenwaks Z. Ovulation induction for assisted reproduction. J reprod Med 1989;34:108-14.

21. Flood JT, Muasher SJ, Simonetti S, Kreiner D, Acosta AA, Rosenwaks Z. Comparison between laparoscopically and ultrasonographically guided transvaginal follicular aspiration methods in an in vitro fertilization program in the same patients using the same stimulation. J In Vitro Fert Embryo Transf 1989;6:180-5.

22.Rede Latino-americana de Reprodução Assistida. Manual de Procedimentos Laboratório de Reprodução Assistida. Santiago do Chile; 1998.

23. Weigert M, Hofstetter G, Kaipl D, Gottlich H, Krischker U, Bichler K, et al. The effect of smoking on oocyte quality and hormonal parameters of patients undergoing in vitro fertilization-embryo transfer. J Assist Reprod Genet 1999;16:287-93.

24. El-Nemr AL, Al-Shawaf T, Sabatini L, Wilson C, Lower AM, Grudzinskas JG. Effect of smoking on ovarian reserve and ovarian stimulation in in-vitro fertilization and embryo transfer. Hum Reprod 1998;13:2192-8.

25.Harrison KL, Breen TM, Hennessey JF. The effect of patient smoking habit on the outcome of IVF and GIFT treatment. Aust N Z J Obstet Gynaecol 1990;30:340-2.

26. Van Voorhis BJ, Syrop CH, Hammitt DG, Dunn MS, Snyder GD. Effects of smoking on ovulation induction for assisted reproductive techniques. Fertil Steril 1992;58:981-5.

27. Hughes EG, YoungLai EV, Ward SM. Cigarette smoking and outcomes of in-vitro fertilization and embryo transfer: a prospective cohort study. Hum Reprod 1992;7:358-61.

28. James VH. An hypothesis on the association between maternal smoking and dizygotic twinning. Hum Reprod 1997;12:1391-2. 